\title{
Simulated Analysis of Dielectric Properties of Heterogeneous Materials
}

\author{
Biao YANG ${ }^{1,2, a}$, Shili WANG ${ }^{1, b}$ and Jinhui PENG $^{2, \mathrm{c}}$ \\ ${ }^{1}$ Faculty of Information Engineering and Automation, Kunming University of Science and \\ Technology, Kunming 650500, China \\ ${ }^{2}$ Key Laboratory of Unconventional Metallurgy, Ministry of Education, Kunming University of \\ Science and Technology, Kunming 650093, China. \\ aybiaocn@163.com¹, ${ }^{\mathrm{b}} 2216108250 @ q q . c o m,{ }^{\mathrm{c}} \mathrm{Jhpeng}$ _ok@Yeah.net
}

\begin{abstract}
The present paper reports the results of a numerical analysis of two phases lossless heterogeneous materials based on a three dimensional (3-D) random dielectric mixture. The effective permittivity of a 3-D dielectric materials is calculated by the S-parameter retrieval method and finite element method. The calculated effective permittivity is in good agreement with theoretical models by compared with Bruggeman formula and Coherent potential formula, showing that the volume fraction of inclusions is increasing, the effective permittivity increases with radiation frequency and appear an imaginary part taking on the dielectric loss properties.
\end{abstract}

\section{Introduction}

Heterogeneous materials containing two phases that are conductive and dielectric are present in a wide variety of man-made materials and in nature. Experimental and theoretical studies of heterogeneous dielectric materials have been in a continuous state of development since the initial work of Maxwell. A number of models such as transmission line modeling or matrix (TLM) method [1], effective medium theories, bounding methods, percolation theory, random walks, hopping model, Fourier expansion, FDTD [2], RC network method, have been documented that dealt with modeling of electromagnetic properties of heterogeneous materials. Many interesting phenomena, e.g. percolation phenomenon [3], local electric field enhancement phenomenon [4], are presented. most studies focused on frequency dependent dielectric properties of specified materials utilized experimental method. Doyle [5] developed an iterative multiple approach to simulate the dielectric properties of heterogeneous particulate materials with arbitrary microstructure, and found that effective permittivity are frequency dependent. Effects of frequency dependent of two phase composite materials were investigated in a two-dimensional formulation by Sun [6]. Inspired by these works, we report here three-dimensional model of two phase heterogeneous materials for the simulation of electromagnetic properties of heterogeneous materials and compare this with the results of the 2D based on FEM and S parameters retrieval algorithm. It shows that the effective permittivity of heterogeneous

* Corresponding author:ybiaocn@163.com 
materials is in good agreement with the results of the Bruggeman formula and CP formula, meanwhile, the real and imaginary part of effective permittivity of modeled heterogeneous materials are frequency dependent even when both inclusions and host are lossless materials.

\section{Modeling and Simulation Calculation}

It is assumed that the heterogeneous materials consists of host phase, its permittivity $\varepsilon_{\mathrm{e}}$ $=1$, and cuboid-shaped inclusions phase, its permittivity $\varepsilon_{\mathrm{i}}=8$ and their side-length of these particles is $1.5 \mathrm{~mm}(<\lambda / 10$ for accuracy). The microstructure model of composite materials and its effective permittivity model are shown in Fig. 1. The composite has successfully been shown to interact with electromagnetic radiation in the same manner as would homogeneous materials with equivalent $S$ parameters [7], as shown in Fig.1(b). Moreover, it is assumed that the size of waveguide is $45 \mathrm{~mm} \times 35 \mathrm{~mm}$, and at the walls of waveguide, a perfect conducting condition is utilized. Distances from the right, left port to the composite, $\boldsymbol{I}_{\mathrm{i}}, \boldsymbol{I}_{\mathrm{j}}$, are both equal to $15 \mathrm{~mm} . \boldsymbol{\beta}_{\mathrm{i}}, \boldsymbol{\beta}_{\mathrm{j}}$ are propagation constant on left port (port i) and right port (port j), respectively.
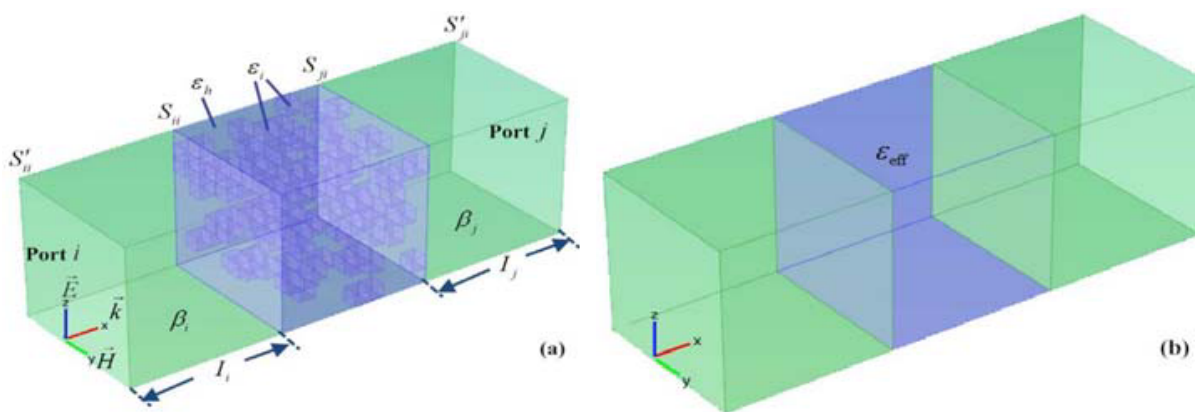

Fig.1 calculation model and (b) effective medium model

In order to characterize the electromagnetic properties of composite, S-parameter retrieval methods [8] are adopted. The S-parameter retrieval procedure operates as follows:

i) Obtain $\mathrm{S}$ parameters from electric fields. Set the composite interfaces as reference phases [9].

$$
\begin{array}{r}
S_{i i}=\left|S_{i i}^{\prime}\right| \exp \left(j\left(2 \beta_{i} I_{i}+\phi_{i i}^{\prime}\right)\right) \\
S_{j i}=\left|S_{j i}^{\prime}\right| \exp \left(j\left(\beta_{i} I_{i}+\beta_{j} I_{j}+\phi_{j i}^{\prime}\right)\right),
\end{array}
$$

where S' can be obtained from electric fields on ports by the following equation [10]

$$
S_{i i}^{\prime}=\int_{\text {porti }} \stackrel{r}{E_{c}}-\stackrel{r}{E_{i}} \cdot \stackrel{r}{E_{i}} d A_{i} / \int_{\text {porti }}^{\stackrel{r}{E_{i}}} \cdot \stackrel{r}{E_{i}} d A_{i}
$$




$$
S_{j i}^{\prime}=\int_{\text {port }} \underset{j}{\stackrel{r}{E}} \cdot \stackrel{r}{E_{j}} \cdot d A_{j} / \int_{\text {port }}^{\underset{j}{E}} \underset{j}{r} \cdot \stackrel{r}{E}{ }_{j} d A_{j}
$$

where $\stackrel{r}{E}$ and $\stackrel{r}{E}_{j}$ are the excitation electric field on port $\mathrm{i}$ and port $\mathrm{j}$ respectively; $\stackrel{\mathrm{r}}{{ }_{c}}$ is the computed electric field on the port $i$ and port $j$, which is equal to the sum of the excitation field and reflected field.

ii) Using the $S$ parameters, we obtain the effective permittivity $\mathcal{E}_{\text {eff }}$ according to the following equation:

$$
\varepsilon_{\text {eff }}=n / Z
$$

where $n, z$ is the refractive index, wave impedance of the composite, respectively. These parameters of heterogeneous materials can be obtained in terms of the $\mathrm{S}$ parameters as follows [8]:

$$
\begin{aligned}
& n=\frac{1}{k d} \cos ^{-1}\left[\frac{1}{2 S_{21}}\left(1-S_{11} S_{22}+S_{21}^{2}\right)\right] \\
& z=\frac{\left(T_{22}-T_{11}\right) \mu \sqrt{\left(T_{22}-T_{11}\right)^{2}+4 T_{12} T_{21}}}{2 T_{21}},
\end{aligned}
$$

where $\mathrm{k}$ is the propagation constant in free space; $\mathrm{d}$ is the thickness of the composite. The value for $\mathrm{z}$ determined with $\operatorname{Re}\left(\varepsilon_{\text {eff }}\right)>0$. T parameters can be expressed in terms of $\mathrm{S}$ parameters as follows:

$$
\begin{gathered}
T_{11}=\frac{\left(1+S_{11}\right)\left(1-S_{22}\right)+S_{21} S_{12}}{2 S_{21}}, T_{12}=\frac{\left(1+S_{11}\right)\left(1+S_{22}\right)-S_{21} S_{12}}{2 S_{21}}, \\
T_{21}=\frac{\left(1-S_{11}\right)\left(1-S_{22}\right)-S_{21} S_{12}}{2 S_{21}}, T_{22}=\frac{\left(1-S_{11}\right)\left(1+S_{22}\right)+S_{21} S_{12}}{2 S_{21}} .
\end{gathered}
$$

where $f_{i}$ is the excitation frequency and $\mathrm{c}$ is the speed of light in free space. The results of the computation are validated by compared with the exponential mixing formula [11] in next section. 


$$
\frac{\varepsilon_{e f f}-\varepsilon_{e}}{\varepsilon_{e f f}+2 \varepsilon_{e}+v\left(\varepsilon_{e f f}-\varepsilon_{e}\right)}=f \frac{\varepsilon_{i}-\varepsilon_{e}}{\varepsilon_{i}+2 \varepsilon_{e}+v\left(\varepsilon_{e f f}-\varepsilon_{e}\right)},
$$

where $f$ is the volume fraction of inclusions, $v=0$ for Maxwell Garnett formula, and $v=1$ for the Bruggeman formula.

\section{Results and Discussion}

\subsection{The Effects of Volume Fractions on S Parameters}

To investigate the interaction of electromagnetic filed with particle inclusion, random and periodic particle inclusions embedded on the host material have been calculated for two cases of given volume fractions: $10 \%$ and $50 \%$. The transmission coefficients $\mathrm{S}_{21}$ of random and periodic models with volume fractions are shown in Fig. 2 (a) and (b).
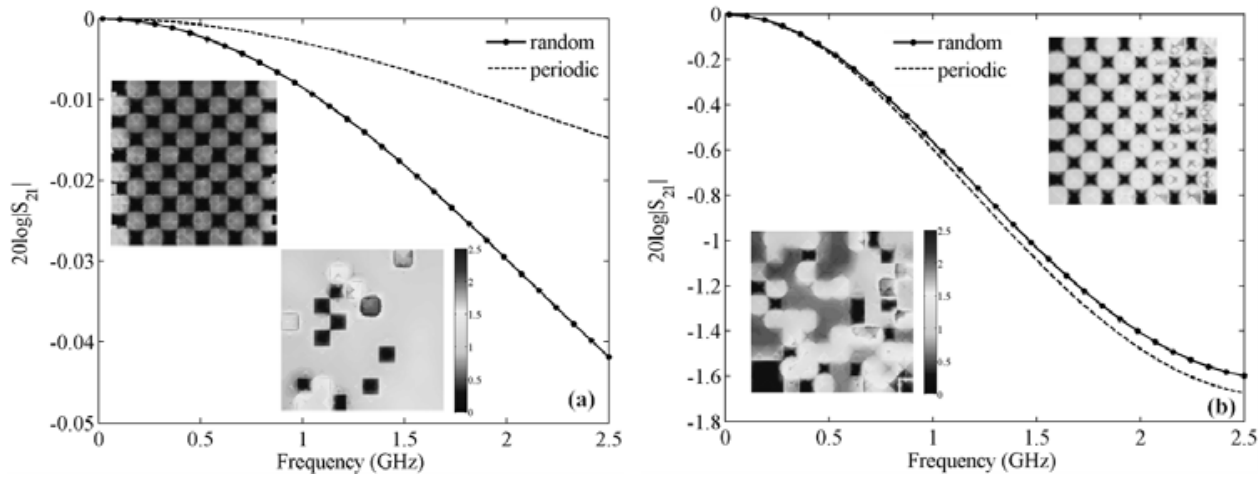

Fig. 2 The transmission coefficient filled with random and periodic particle inclusion of volume

$$
\text { fraction: (a) } 10 \% \text {, (b) } 50 \%
$$

At two cases: volume fraction equal to $10 \%$ and $50 \%$ shown in Fig. 2(a) and (b) , differences of $\mathrm{S}_{21}$ between random and periodic models are 0.03 and 0.1 . Besides, these differences increase with the frequency rising. The illustrations of Fig. 2 show the distributions of electric field for random and periodic models with volume fraction setting as $10 \%$ and $50 \%$ at $2 \mathrm{GHz}$. It can be seen that local field enhancement appears in the random model. This phenomenon is in good agreement with the results of Yang et al. [4]. This phenomenon is explained that when the concentration of inclusions is increased, the average distance between particles decrease, which the effects of volume fractions on $\mathrm{S}$ parameters and local field distribution have been highlighted. 

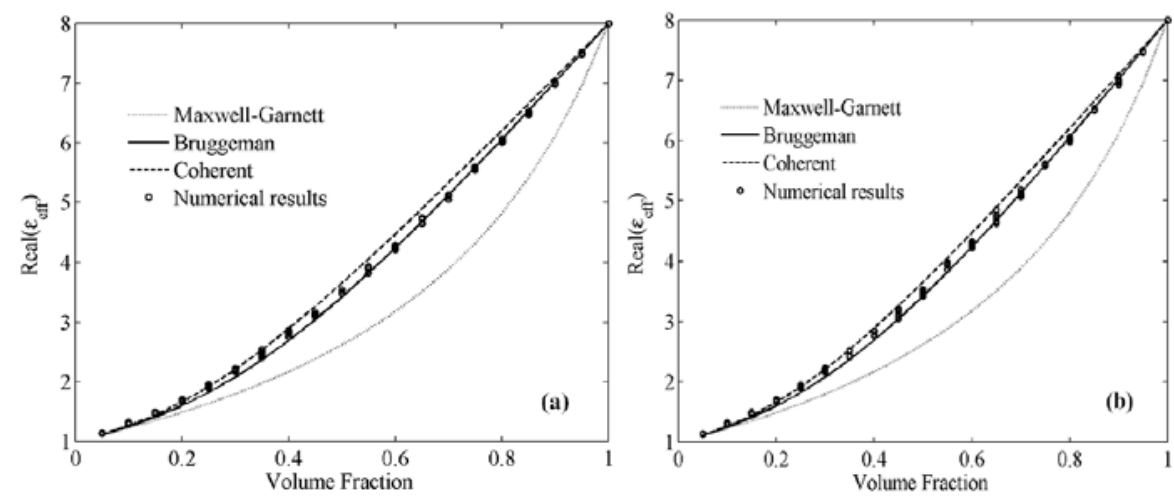

Fig. 3 The effective permittivity as a function of volume fractions at different frequenc: (a)

$20 \mathrm{MHz}$, (b) $2 \mathrm{GHz}$

\subsection{The Influences of Volume Fractions on Effective Permittivity}

The effective permittivity of random and periodic models have been simulated by using the S parameters retrieval methods at $20 \mathrm{MHz}$ and $2 \mathrm{GHz}$ in which five random distributed topological structures have been calculated for each volume fraction, respectively. As shown in Fig. 3(a) and 3(b), the standard deviations of effective permittivity are $21.2 \%$, $2.2 \%$ and $1.2 \%$ compared with Maxwell-Garnett, Bruggeman formula and Coherent potential at $20 \mathrm{MHz}$. Whereas the standard deviations are $21.4 \%, 2.3 \%$ and $1.4 \%$ at $2 \mathrm{GHz}$. It shows that the effective permittivity of composites computed in this paper are good agreement with Eq. (11) at low volume fractions. The discrepancy of effective permittivity have been enlarged when the volume fraction and frequency increase. Therefore, the effective permittivity will be subject to the random distribution of particles and the frequency of electromagnetic fields.

\subsection{Relations Between Effective Permittivity and Frequency}

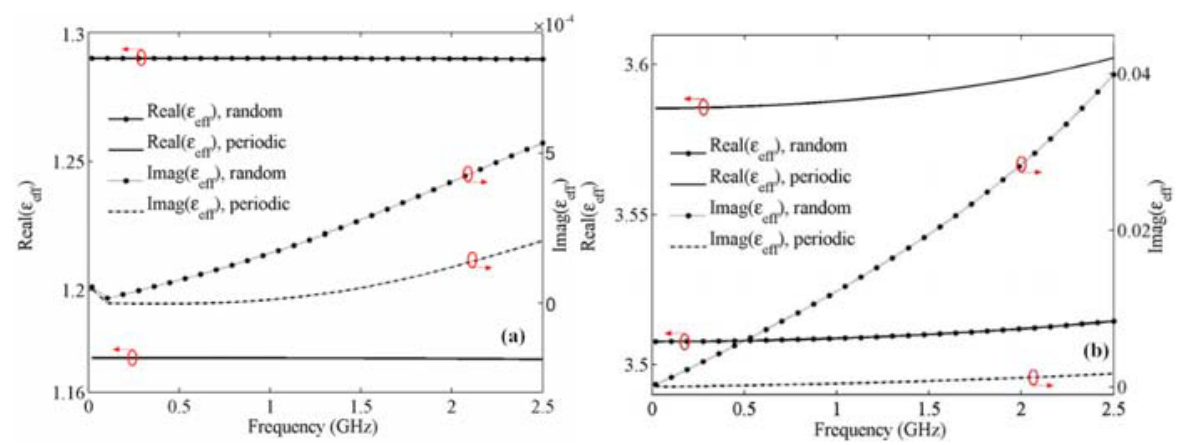

Fig. 4 The effective permittivity of heterogeneous materials as function of frequency with volume fraction

(a) $10 \%$, (b) $50 \%$

The relation between the effective permittivity and the frequency is illustrated in Fig.4, which includes the volume fractions of the heterogeneous materials are $10 \%$ and $50 \%$. It can be seen from Fig. 4(a) that the real part of effective permittivity of periodic and random 
model unchanged with frequency, moreover the imaginary part hardly change, approximate to $5 \times 10-4$. However, according to Fig. 4(b), with increasing frequency, it is apparent that the effective permittivity of periodic and random model will increase at volume fraction equal to $50 \%$. This phenomenon has been reported by Doyle et al. [5], which claimed that it is resulted to Mie resonances. We find interestingly that the imaginary part of effective permittivity changes more magnitude at the random model than at the periodic model, reaching to 0.04 , which is counterintuitive due to both inclusions and host are lossless materials.

\section{Conclusion}

The dielectric properties of composite materials is very important in electromagnetic application fields. The effective permittivity of two phase composite materials has been computed in this paper. Results show that the effective permittivity of model is in good agreement with Maxwell Garnett rule at low volume fractions; the discrepancy of effective permittivity have been enlarged when the volume fraction and frequency increase; Effective permittivity as a function of the volume fraction of inclusions; the real part and imaginary part of effective permittivity of random model increases with frequency even if both the inclusions and host materials are frequency independent and lossless.

\section{Acknowledges}

This work is partially supported by project (No. KKSY201503006) Scientific Research Foundation of Kunming University of Science and Technology, project (51090385) the Major Program of National Natural Science Foundation of China.

\section{References}

1. C. Christopoulos, The Transmission-Line Modeling (TLM) Method in Electromagnetics, First ed., University of Nottingham, UK, 2006.

2. K.S. Yee, Numerical solution of initial boundary value problem involving Maxwell's equations inisotropic media, IEEE Transactionson Antennas and Propagation, 14 (1966) 302-307.

3. M. Huang, J.J. Yang, Z. Xiao, J. Sun, J.H. Peng, Modeling the dielectric response in heterogeneous materials using 3D RC networks, Modern Physics Letters, 23 (3009) 3023-3033.

4. J.J. Yang, M. Huang, J.H. Peng, Electromagnetic properties of heterogeneous materials and the local electric field enhancement effects, Material Review Research, 23 (2009) 1-5.

5. T.E. Doyle, D.A. Robinson, S.B. Jones, K.H. Warnick, B. L. Carruth, Modeling the permittivity of two-phase media containing monodisperse spheres: Effects of microstructure and multiple scattering, Physical Review B, 76(2007) 054203.

6. J. Sun, J.H. Peng, The simulation of the frequency-dependent effective permittivity for composite materials, 9th International on Antennas, Guangzhou: IEEE Press, 2010: 701-704.

7. R.A. Shelby, D.R. Smith, S. Schultz, Experimental verification of a negative index of refraction, SCience, 292 (2001) 77-79.

8. D.R. Smith, D.C. Vier, T. Koschny, C.M. Soukoulis, Electromagnetic parameter retrieval from inhomogeneous metamaterials, Physical Review E, 71(2005) 36617. 
9. W. Weir, Automatic measurement of complex dielectric constant and permeability at microwave frequencies, Proceedings of the IEEE, 62(1974) 33-36.

10. Information on http://www.comsol.com, COMSOL multiphysics user's guide, 2009.

11. A.H. Shivola, Self-consistency aspects of dielectric mixing theories, IEEE Transactions on Geoscience and Remote Sensing, 27(1989) 403-415. 